\title{
A ergonomia no ambiente de trabalho dos enfermeiros do samu: uma visão da
}

\section{enfermagem}

Ergonomics in the work environment of samu nurses: a view of nursing

Ergonomía en el entorno laboral de las enfermeras samu: una visión de la enfermería

Recebido: 28/12/2020 | Revisado: 06/01/2021 | Aceito: 13/01/2021 | Publicado: 15/01/2021

Marciele de Lima Silva

ORCID: https://orcid.org/0000-0003-2827-5316

Instituto de Educação Superior da Paraíba, Brasil E-mail: marcieledelsilva@gmail.com

Mariana Pereira Barbosa Silva

ORCID: https://orcid.org/0000-0003-0852-8099

Universidade Estadual do Piauí, Brasil

E-mail: marianapbsilvaa@gmail.com

Airton César Leite

ORCID: https://orcid.org/0000-0001-7184-8488

Centro Universitário Santo Agostinho, Brasil E-mail: ainton.cesar2014@gmail.com

Elton Filipe Pinheiro de Oliveira

ORCID: https://orcid.org/0000-0003-0930-859X

Universidade Federal do Piaú, Brasil

E-mail: helton-oliver@ hotmail.com

Ana Rachel Cavalcante Araújo Fernandes

ORCID: https://orcid.org/0000-0003-0913-3451

Universidade de Fortaleza, Brasil

E-mail: rachel_cavalcante@hotmail.com

Diana Nogueira Villa Jatobá

ORCID: https://orcid.org/0000-0003-4346-8335

Centro Universitário UNINOVAFAPI, Brasi

E-mail: devilla@hotmail.com

Francinalda Pinheiro Santos

ORCID: https://orcid.org/0000-0003-1222-7854

Instituto de Ensino Superior Múltiplo, Brasil

E-mail: franfilho2@hotmail.com

Mara Cléssia de Oliveira Castro

ORCID: https://orcid.org/0000-0002-1808-7963 Centro Universitário UNINOVAFAPI, Brasil

E-mail: maraclessia@hotmail.com

Vinícius de Sousa Martins

ORCID: https://orcid.org/0000-0002-9443-922X

Instituto de Ensino Superior Múltiplo, Brasil

E-mail: vinicius.smartins@yahoo.com.br

Aclênia Maria Nascimento Ribeiro

ORCID: https://orcid.org/0000-0002-5582-9663 Universidade Federal do Piauí, Brasil

E-mail: aclennya@hotmail.com

Layanne Cavalcante de Moura

ORCID: https://orcid.org/0000-0003-2781-1076

Centro Universitário UNIFACID, Brasil

E-mail: layannecavalcante@hotmail.com

Cicera Leiane Sampaio Rodrigues

ORCID: https://orcid.org/0000-0001-8038-1242

Faculdade de Medicina Estácio Juazeiro do Norte, Brasil

E-mail: leianesampaio01@gmail.com

Ericka Costa de Azevedo Freire

ORCID: https://orcid.org/0000-0003-1073-4406

Instituto de Educação Superior da Paraíba, Brasil

E-mail: ericka_azvedo@hotmail.com

\section{Resumo}

A ergonomia é considerada uma das principais estratégias na prevenção de algumas doenças laborais, e é utilizada com o objetivo de identificar situações desfavoráveis durante a realização de atividades nos ambientes de trabalho, a fim de reduzir os riscos sem prejuízo do desempenho profissional. Nesse contexto, o Serviço de Atendimento Móvel 
de Urgência constantemente expõe os seus trabalhadores de enfermagem a essas circunstâncias, devido às especificidades dos serviços de assistência direta ao paciente fora do ambiente hospitalar, visando à manutenção da vida em situação de urgência e emergência antes da chegada a uma instituição de atendimento especializado. O objetivo deste estudo foi avaliar artigos científicos da área de saúde que abordam as condições de trabalho no SAMU e refletir acerca das melhorias no posto de trabalho do profissional de enfermagem no SAMU. Trata-se de uma revisão bibliográfica realizada nas bases de dados científicos Medline, Lilacs, Scielo e BVS. Para seleção dos artigos foram utilizados, os descritores: Ergonomia física, Enfermagem, SAMU e Atendimento Pré-hospitalar. Os resultados demonstraram que os trabalhadores de enfermagem estão expostos a inúmeros riscos ocupacionais causados por fatores químicos, físicos, mecânicos, biológicos, ergonômicos e psicossociais, é importante que os mesmos sejam informados e treinados para evitar problemas de saúde, e métodos de controle devem ser instituídos para prevenir acidentes. Em vista disso, conclui-se que este estudo é relevante para a literatura no tocante ao incentivo do conhecimento teórico-prático acerca da ergonomia no ambiente hospitalar.

Palavras-chave: Ergonomia física; Enfermagem; SAMU; Atendimento pré-hospitalar.

\begin{abstract}
Ergonomics is considered one of the main strategies in the prevention of some occupational diseases, and it is used with the objective of identifying unfavorable situations when carrying out activities in the work environment, in order to reduce risks without prejudice to professional performance. In this context, the Mobile Emergency Care Service constantly exposes its nursing workers to these circumstances, due to the specificities of direct patient care services outside the hospital environment, aiming at maintaining life in an urgent and emergency situation before arrival to a specialized service institution. The aim of this study was to evaluate scientific articles in the health field that address working conditions at SAMU and reflect on improvements in the nursing professional's work at SAMU. This is a bibliographic review carried out in the scientific databases Medline, Lilacs, Scielo and VHL. The following descriptors were used to select the articles: Physical ergonomics, Nursing, SAMU and Pre-hospital care. The results showed that nursing workers are exposed to numerous occupational risks caused by chemical, physical, mechanical, biological, ergonomic and psychosocial factors, it is important that they are informed and trained to avoid health problems, and control methods must be instituted to prevent accidents. In view of this, it is concluded that this study is relevant to the literature with regard to encouraging theoretical and practical knowledge about ergonomics in the hospital environment.
\end{abstract}

Keywords: Physical ergonomics; Nursing; SAMU; Pre-hospital care.

\title{
Resumen
}

La ergonomía se considera una de las principales estrategias en la prevención de algunas enfermedades profesionales, y se utiliza con el objetivo de identificar situaciones desfavorables a la hora de realizar actividades en el entorno laboral, con el fin de reducir los riesgos sin perjuicio del desempeño profesional. En este contexto, el Servicio Móvil de Emergencias expone constantemente a sus trabajadores de enfermería a estas circunstancias, debido a las especificidades de los servicios de atención directa al paciente fuera del ámbito hospitalario, con el objetivo de mantener la vida en una situación de urgencia y emergencia antes de la llegada a una institución de servicios especializada. El objetivo de este estudio fue evaluar artículos científicos en el campo de la salud que abordan las condiciones laborales en SAMU y reflexionan sobre las mejoras en el trabajo del profesional de enfermería en SAMU. Se trata de una revisión bibliográfica realizada en las bases de datos científicas Medline, Lilacs, Scielo y VHL. Para la selección de los artículos se utilizaron los siguientes descriptores: ergonomía física, enfermería, SAMU y atención prehospitalaria. Los resultados mostraron que los trabajadores de enfermería están expuestos a numerosos riesgos laborales ocasionados por factores químicos, físicos, mecánicos, biológicos, ergonómicos y psicosociales, es importante que estén informados y capacitados para evitar problemas de salud, y se deben implementar métodos de control. instituido para prevenir accidentes. Ante esto, se concluye que este estudio es relevante para la literatura en lo que se refiere a fomentar el conocimiento teórico y práctico sobre ergonomía en el ámbito hospitalario.

Palabras clave: Ergonomía física; Enfermería; SAMU; Atención prehospitalaria.

\section{Introdução}

O Serviço de Atendimento Móvel de Urgência - SAMU foi regulamentado no Brasil em 2002 por meio da Portaria $\mathrm{n}^{\circ}$ 2.048/GM. O SAMU é considerado parte de uma rede de atenção às urgências, e tem como objetivo organizar o fluxo assistencial e prestar serviços à população, seja no tocante aos primeiros primários de urgência, como no transporte adequado, rápido às vítimas acometidas por agravos à saúde de várias naturezas com a finalidade de reduzir a morbimortalidade das mesmas. Para tal atendimento, sua equipe é composta de uma equipe multiprofissional, composta por condutores, profissionais da enfermagem, da medicina e da área administrativa (Melo et al., 2016). 
Diante de tantas demandas, o profissional se expõe a riscos, seja devido ao estresse gerado pelo impacto físico das atividades, seja pelo impacto emocional ao qual estão expostos no ambiente laboral. Ora, a equipe que atua na urgência móvel está sujeita a uma carga de trabalho exaustiva, relacionada à complexidade do estado da vítima e a gravidade em que a encontra no momento do socorro, além do deslocamento da ambulância, do tráfego diário em meio ao trânsito exaustivo, entre outros (Worm et al., 2016).

Por sua vez, o ambiente laboral abrange um conjunto de fatores que causam riscos ao profissional, comprometendo sua saúde a curto ou longo prazo (Gomes, 2016). Os profissionais do SAMU, são expostos à riscos biológicos, físicos, químicos, psicossociais e ergonômicos (Moura \& Rezende, 2017).

Atualmente, a procura por melhor qualidade laboral, está associada à maior produtividade, que por sua vez, depende diretamente da segurança que o profissional possui no desenvolvimento de suas atividades. Além da segurança, a ergonomia é de extrema importância para garantir a saúde do trabalhador e sua aplicação vem se intensificando nos ambientes de trabalho, de modo a prevenir o profissional de riscos que comprometam sua saúde. Pelo exposto, a ergonomia possui grande impacto para o profissional de enfermagem em atendimento móvel de urgência (Bertoldi, 2018). Vale ressaltar que os riscos ergonômicos levam muitos profissionais ao afastamento do trabalho, devido a incapacidade, caso medidas preventivas não sejam tomadas.

Considerando que os profissionais que atuam no atendimento pré-hospitalar - APH deparam-se com diversos problemas como a falta de treinamento adequado, a falta de segurança da equipe quando precisa atuar em locais perigosos, espaço físico limitado para realização de procedimentos, por diversas vezes com a ambulância em movimento, limitações também para técnicas invasivos e descarte de resíduos e materiais perfuro-cortantes entre outros (Portela et al., 2018). Existem ainda os problemas relacionados ao peso do paciente, seja para colocá-lo na maca, ou para deslocar a maca até a ambulância ou do transporte até o hospital, peso dos cilindros de oxigênio (Andrade, 2017).

Sabe-se que é necessário identificar os riscos ocupacionais aos quais a equipe de enfermagem do APH estão expostas, para que melhorias no ambiente de trabalho sejam feitas a fim de garantir um desenvolvimento das atividades laborais seguras e livres de danos à saúde dos mesmos (Souza, Pinheiro, \& Moésia, 2016).

O interesse pela temática surgiu ao se observar a ausência da ergonomia entre os profissionais de enfermagem que atuam no SAMU. Partindo do pressuposto, emergiu-se a pergunta norteadora deste estudo foi: Como a literatura aborda a ergonomia no serviço de atendimento móvel em saúde? Diante deste cenário, a finalidade deste estudo foi avaliar artigos científicos da área de saúde que abordem as condições de trabalho no SAMU e refletir acerca das melhorias no posto de trabalho do profissional de enfermagem no SAMU.

\section{Metodologia}

Trata-se de uma revisão de literatura, realizada a partir de artigos científicos relacionados à temática explorada. Para Prestes (2003), a revisão de literatura procura resolver algum problema ou adquirir conhecimentos a partir de informações provenientes de material. Fachin (2003), reforça que a mesma diz respeito ao conjunto de conhecimento humano resumidos nas obras, tendo como finalidade conduzir o leitor a determinado assunto e proporcionar a produção, armazenamento, reprodução, utilização e comunicação das informações coletadas para o desenvolvimento da pesquisa.

O material do estudo foi coletado através da busca online a partir do Google acadêmico com os seguintes descritores: Ergonomia, Enfermagem, Serviço Médicos de Emergência. Para seleção dos artigos foram utilizados os seguintes critérios de inclusão: textos completos, idioma português, publicado no período de 2015 a 2020. Os critérios de exclusão foram: artigos que não se adequassem ao tema proposto. 


\section{Resultados e Discussão}

A nível mundial, o termo ergonomia está presente desde o período posterior à II guerra mundial, no entanto, para a fundamentação teórica da disciplina de ergonomia, as pesquisas tiveram início logo após, tendo assim, aproximadamente, menos de 70 anos todo este percurso de conhecimento. Nesse contexto, a ergonomia no Brasil foi normatizada no ano de 1990 , sendo o setor da saúde, considerado uma área bastante complexa, composto de procedimentos dinâmicos e variadas atividades singulares. Assim, ao considerar o serviço de atendimento móvel de urgência (SAMU), leva-se em conta a assistência préhospitalar, cujos atendimentos são feitos em automóveis específicos para o translado de pacientes, porém com espaços reduzidos o que não favorece o bom desempenho do trabalho do profissional de saúde (Bertoldi, 2018).

Para Gomes (2016), as alterações nas características epidemiológicas nos anos 90, foram desencadeadas pela elevação no índice de morbimortalidade do Brasil. Nesse sentido, diversos fatores contribuíram para a criação de políticas no âmbito do Ministério da Saúde, visando melhoria no atendimento às urgências, proporcionando uma assistência mais fidedigna à população, como por exemplo: aumento dos casos de violência urbana, acidentes, assim como a insuficiente infraestrutura dos serviços de emergência.

Assim, a partir da Portaria n ${ }^{\circ}$ 2.048, de 2002, surgiu o SAMU. E no ano de 2003 surgiram duas novas Portarias: a de $n^{\circ} 1.863$ que instituiu a Política Nacional de Atenção às Urgências e a nº 1.864 que estabeleceu o componente pré-hospitalar móvel em todo o território brasileiro (Brasil, 2002).

Os serviços de atendimento pré-hospitalar móvel são compostos com equipe de profissionais variados que são distribuídos conforme o modelo de atendimento, sendo geralmente formados por médicos, enfermeiros, técnicos/ auxiliares de enfermagem, condutores de veículo, pilotos e técnicos de operações especiais. Esses profissionais atuam em equipe com o objetivo de fornecer uma assistência ágil e com bom desfecho para o paciente (Brasil, 2013).

Conforme a Norma Regulamentadora $n^{\circ} 9$ do Ministério do Trabalho e Emprego - MTE, os agentes físicos, químicos e biológicos presentes no ambiente laboral, são considerados riscos ambientais, devido à possibilidade de provocar algum dano à saúde do profissional. É importante ressaltar ainda, que existem os riscos ergonômicos, psicossociais, mecânicos e de acidentes. Os ergonômicos e psicossociais são provenientes da organização e administração do trabalho, e como por exemplo, podemos citar: o uso de equipamentos, máquinas e mobiliários de maneira inadequados, o que pode ocasionar o desenvolvimento de posturas e posições incorretas; lugares com pouca iluminação; atividades em excesso; trabalho em período noturno; entre outros (Portela et al., 2018).

Os profissionais que atuam no SAMU, comumente são envolvidos em situações onde a assistência ao paciente é realizada em locais hostis, adversos e imprevisíveis, onde a prioridade do atendimento constitui-se na avaliação da segurança da cena, visto que os socorristas, geralmente, são vulneráveis a acidentes ocupacionais, resultantes de elementos externos vindos do lugar onde é feito o atendimento. (Gomes, 2016). Nesse cenário, os membros da equipe do Atendimento PréHospitalar (APH) móvel estão sujeitos aos mais diversos riscos, sendo a maioria dos acidentes relacionados aos perfurocortantes, seguido por acidentes provenientes de riscos ergonômicos, além de outros riscos (Melo et al., 2016).

É importante enfatizar, que o conhecimento do enfermeiro necessita também ser relacionado ao uso de equipamentos de proteção individual (EPIs), e critérios de segurança, como a Norma Regulamentadora 32 (NR-32), que regulamenta o trabalho em saúde. Portanto, é fundamental o conhecimento acerca das Precauções Padrão (PP), que se constitui no agrupamento de regras relacionadas às práticas seguras e atividades mediante o risco biológico, que é consideravelmente elevado no Atendimento Pré-Hospitalar, devido aos múltiplos ambientes laborais (Almeida \& Álvares, 2019).

Quanto aos riscos ocupacionais que são evidentes nas atividades da equipe atuante nos serviços de urgência e emergência, é de essencial importância compreender a relação do processo saúde-doença do trabalhador e, a partir disso, criar 
estratégias que visem aprimorar as circunstâncias de trabalho, favorecendo assim, a promoção da saúde e prevenção de agravos, bem como o manejo e redução/eliminação dos riscos e dos problemas de saúde da equipe. Locais de trabalho insalubres ou inadequados, falta de materiais de trabalho, e necessidade de improvisos, provocam desgaste físico e mental, perda de tempo e insegurança nas atividades desenvolvidas, sendo responsável por um panorama de estresse (Gomes, 2016).

Prates (2018), enfatiza que diversas situações associadas às condições de trabalho e que visam o aumento da produtividade, porém com reduzida preocupação com o profissional, costumam gerar problemas de saúde e estão envolvidos no processo de adoecimento dos enfermeiros. Desse modo, no que se refere ao trabalho da enfermagem, os trabalhadores no decorrer do desenvolvimento de suas atividades, estão vulneráveis a vários riscos ocupacionais causados por fatores variados, incluindo os ergonômicos, que podem ocasionar doenças ocupacionais e acidentes de trabalho. Acredita-se que estes riscos ocupacionais associados às más condições de trabalho se intensificam.

Saçala, Luvizotto, Oselame e Neves (2017), infere que o profissional que faz parte da equipe de atendimento préhospitalar desenvolve atividades que são responsáveis por um grande desgaste físico, tudo isso, ocasionado, entre outros fatores, pelas características do próprio ambiente de trabalho. Esses profissionais se envolvem diariamente com diversas situações no intuito de prestar assistência às vítimas, e se verem obrigados a se expor aos mais variados ambientes naturais, enfrentar locais de acesso difícil, e que representam riscos, muitas vezes, para a própria vida.

A exposição a estes locais são contínuos e são aumentados pelo ritmo intenso e excessivas jornadas de trabalho que acarretam redução na produtividade, bem como, uma queda na qualidade de vida. Em decorrência dessa exposição contínua, também pode ocorrer afastamento para tratamento de saúde e invalidez, faltas ao trabalho, o que ocasiona uma redução de funcionários e sobrecarga de trabalho para o restante da equipe. Outro fator que favorece o comprometimento físico dos trabalhadores de saúde que exercem atividades no setor no pré-hospitalar é que estes, geralmente, ignoram o próprio desconforto físico para garantir a sobrevida dos pacientes. Assim, é importante dizer que os distúrbios osteomusculares podem estar associados com a falta organizacional no trabalho, ou seja, aumento da jornada de trabalho, horas extras excessivas, ritmo acelerado e déficit de profissionais no setor (Saçala, Luvizotto, Oselame \& Neves, 2017).

No estudo realizado por Worm et al. (2016) a equipe de enfermagem do SAMU relatou insatisfação com a desorganização do seu local de trabalho. Já, com relação às lesões físicas, as dores na coluna vertebral e distúrbio do sono, foram relacionados com a má qualidade no desenvolvimento do profissional enfermeiro.

A equipe de enfermagem está exposta a inúmeros riscos ocupacionais, seja os causados por fatores físicos, mecânicos, químicos, biológicos, ergonômicos e psicossociais, que podem causar o desenvolvimento de doenças e acidentes de trabalho. Os profissionais que estão expostos a tais riscos precisam estar capacitados de modo a evitar problemas de saúde (Souza et al., 2016).

O distúrbio do sono foi relatado em três artigos da amostra e está relacionado aos plantões noturnos e a necessidade de conciliar vários empregos. Ainda existem as dificuldades relacionadas às condições desconfortáveis do alojamento da equipe, falta de reconhecimento profissional, de recursos humanos, baixa remuneração, dificuldade para chegar ao local do evento (Dias et al., 2016).

\section{Conclusão}

A atuação dos enfermeiros no SAMU é marcada por muitos desafios que causam desgastes e estresse, por outro lado, suas ações assistenciais exercem papel importante no SAMU/APH. 
O presente estudo possibilitou identificar que os profissionais de APH possuem conhecimento sobre os fatores de risco associados ao ambiente de trabalho, entendendo que a sobrecarga, aliada às falhas na estrutura organizacional, são fatores que podem determinar potenciais riscos ocupacionais.

Conclui-se com esse estudo de revisão integrativa, é relevante para a literatura no tocante ao incentivo do conhecimento teórico-prático acerca da ergonomia no ambiente hospitalar.

Assim, sugere-se que mais pesquisas sobre a temática sejam realizadas, uma vez que existem poucos artigos que relacionem a ergonomia com a atuação do enfermeiro, além da sua importância para que a atuação deste seja mais segura e com qualidade.

\section{Referências}

Almeida, R. B., \& Álvares, A. C. M. (2019). Assistência de enfermagem no serviço móvel de urgência (SAMU): revisão de literatura. Revista De Iniciação Científica Extensão, 2(4), 196-207.

Andrade, I. C. S. (2017). Riscos ocupacionais na prática da equipe de enfermagem no serviço de atendimento móvel de urgência. Monografia. Faculdade Maria Milza, Bahia, Governador Mangabeira, Brasil.

Bertoldi, S. V. P. (2018). Avaliação ergonômica do profissional de enfermagem no serviço privado de atendimento móvel de urgência. Monografia (Especialização). Universidade Tecnológica Federal do Paraná, Paraná, Medianeira, Brasil.

Brasil. Ministério da Saúde. Portaria n. ${ }^{\circ}$ 2048, de 5 de novembro de 2002. Aprova o Regulamento Técnico dos Sistemas Estaduais de Urgência e Emergência. Diário Oficial da União, Brasília, DF, 12 nov. 2002.

Brasil. Ministério da Saúde. Manual Instrutivo da Rede de Atenção às Urgências e Emergências no Sistema Único de Saúde (SUS). 1 ed. Brasília-DF: Ministério da Saúde, 2013. 84 p. 25-26.

Dias, L. P. R., Mendes, R. S., Trigueiro, G. P., Assis, E. V., Feitosa, A. N. A., \& Sousa, M. N. A. (2016). Enfermagem no atendimento pré-hospitalar: papel, riscos ocupacionais e consequências. Revista Interdisciplinar em Saúde, 3(1), 223-236.

Fachin, O. (2003). O fundamento de metodologia ( $5^{\mathrm{a}}$ Ed.). São Paulo: Saraiva.

Gomes, E. A. S. (2016). Condições de trabalho e estresse na equipe do samu: uma revisão integrativa. Trabalho de conclusão de curso. Universidade Federal de Campina Grande, Paraíba, Cuité, Brasil.

Melo, L. S., Barbosa, A. M. G, Araújo, A. M. G., Freitas, M. M. S. M., Lima, M. G. L, \& Melo, L. S. (2016). Riscos ocupacionais no Serviço de Atendimento Móvel de Urgência. Rev. iberoam. educ. investi. Enferm. 6(2):65-72.

Moura, Y., \& Rezende, A. R. (2017). Os riscos da ocupação de socorrista do serviço de atendimento móvel de urgência (SAMU) em uma cidade de pequeno porte. Intercursos Revista Científica, 12(1), 23-33.

Portela, N. L. C., Pedrosa, A. O., Silva, N. S., Ramos, J. S., Silva, R. S., \& Santos, R. T. C. (2018). Riscos ocupacionais entre trabalhadores de um serviço de atendimento móvel de urgência. Reon Facema, 4(2), 986-992.

Prates, P. (2018). As condições de trabalho do (a) enfermeiro(a) na sociedade capitalista e os impactos na sua saúde. Trabalho de conclusão de curso. Universidade Federal do Rio Grande do Sul, Rio Grande do Sul, Porto alegre, Brasil.

Prestes, M. L. M. (2003). A pesquisa e a construção de conhecimento: Do planejamento aos textos, da escola a acadêmica (2 ed.) São Paulo: Répel.

Saçala, R., Luvizotto, J. R., Oselame, G. B., \& Neves, E. B. (2017). Distúrbios osteomusculares relacionados ao processo de trabalho no atendimento préhospitalar. Revista da Universidade Vale do Rio Verde, 15(2), 751-758.

Souza, N. D., Pinheiro, M. B. G. N., \& Moésia, R. V. (2016). Acidentes ocupacionais em profissionais de saúde no atendimento pré-hospitalar. Journal of Medicine and Health Promotion, 1(1), 01-10.

Worm, F. A., Pinto, M. A. O., Schiavenato, D., Ascari, R. A., Trindade, L. L., \& Silva, O. M. (2016). Risco de adoecimento dos profissionais de enfermagem no trabalho em atendimento móvel de urgência. Revista Cuidarte, 7(2), 1279-1287. 